\title{
Numerical theory of accretion flow and jet launching: A study on the galactic center
}

\author{
Salomé Dibi ${ }^{1}$, Samia Drappeau ${ }^{1}$, Sera Markoff ${ }^{1}$ and Chris Fragile ${ }^{2}$ \\ ${ }^{1}$ Astronomical Institute "Anton Pannekoek", University of Amsterdam, \\ Postbus 94249, 1090 GE Amsterdam, the Netherlands \\ email: s.dibi-rousselle@uva.nl \\ ${ }^{2}$ Dept. of Physics \& Astronomy, College of Charleston, \\ 58 Coming Street Charleston, SC 29424, United States
}

\begin{abstract}
We obtained the first spectral predictions from a simulation of the Galactic Center to include radiative processes internally. We performed simulations with and without cooling, with and without spin, and for different initial configurations of the magnetic field, in order to test the effect on jet launching and inner accretion disk characteristics. By exploring parameter space, we will attempt to place new constraints on the controversial question about the presence or not of a jet from Sgr A*, as well as study jet launching in general. We have shown that, as expected, the spin of the $\mathrm{BH}$ affects the structure of the jet. The presence of cooling also strongly influences the inner structure of the accretion disk and therefore affects jet launching. These results show that radiative cooling is not negligible, as is usually assumed for the very underluminous supermassive BH, Sgr A*. On the contrary, the inclusion of cooling has a very visible influence on the accretion disk. Furthermore it creates an important difference in the resulting spectra.
\end{abstract}

Keywords. Sgr A*, MHD, radiation mechanisms: general, plasmas, radiative transfer, diffusion, acceleration of particles.

\section{Modeling Sgr A* with Cosmos ${ }^{++}$}

We present preliminary results of a series of fully relativistic 2D magneto-hydrodynamic simulations (using Cosmos ${ }^{++}$; Anninos et al. 2005) of the accretion flow around a supermassive black hole $(\mathrm{BH})$. The initial state of the simulation is shown in Figure 1. Our

Figure 1. This figure shows the initial $2 \mathrm{D}$ state of the simulation (black and white color scale in density) for a $4.3 \times 10^{6} M_{\odot} \mathrm{BH}$ at $(0,0)$, with an edge-on view of the accretion disk. The black shows the initial concentration of the material inside the accretion disk, set to values that capture the measured accretion rate onto Sgr $\mathrm{A}^{*}$. Together with the matter density, the mesh grid domain used for the computation is represented. Symmetry about the rotation axis of the accretion flow is assumed, as necessary for $2 \mathrm{D}$.

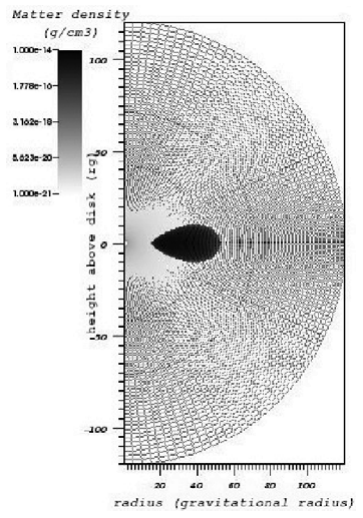

simulations are some of the first that self-consistently include radiative cooling which allows us to compare our predictions to actual observations. In the present work we choose Sgr $A^{*}$ for our first tests as its accretion flow is relatively well constrained compared 
with other sources. We performed simulations with and without cooling, with and without spin, and for different initial configurations of the magnetic field. S. Drappeau (see proceedings article, this volume) has used our output to generate spectra to compare with observational data of the Galactic center. The simulations were all run in $2 \mathrm{D}$, and for 7 complete orbits in order to approximate a steady state.

\title{
2. Results
}

For the models with normalized spin parameter of $a=0.99$, the jet is clearly more relativistic (Lorentz factor 3). Even without spin, plasma is ejected along the axes, but it is less collimated and less relativistic (Fig. 2). For a non-rotating BH $(\mathrm{a}=0)$, the outflow

\begin{abstract}
Figure 2. These panels show the time averaged velocity magnitude of the plasma over the last two orbits for three different simulations of Sgr A*. The models were run with the same initial magnetic field configuration (one poloidal loop crossing the disk, parameter $B_{p}=1$ ) to test the spin (parameter a) and the radiative cooling (parameter $\Lambda$ ). Black and white color scale in velocity from $3 * 10^{7}$ to $3 * 10^{10} \mathrm{~cm} / \mathrm{s}$.
\end{abstract}
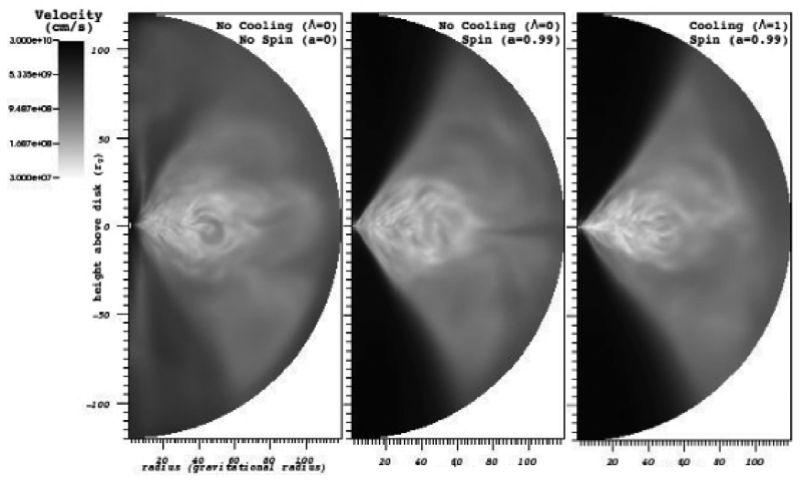

is an order of magnitude slower which is not surprising as the magnetic field strength is also ten times weaker. The presence of cooling strongly influences the inner structure of the accretion disk and therefore affects jet launching. The resulting inner accretion disk is flatter, because in the models without cooling the disk and jet are puffed up by gas pressure due to higher temperatures. These results show that radiative cooling is not negligible, as is usually assumed for the very underluminous supermassive BH, Sgr $\mathrm{A}^{*}$. On the contrary, the inclusion of cooling has a very visible influence on the accretion disk. Furthermore, Samia Drappeau developed a code to generate spectra from our simulations output, taking into account the radiative loss terms included in Cosmos ${ }^{++}$ (bremsstrahlung, synchrotron and inverse Compton). The initial results show significant differences in the spectrum for the models with cooling versus without. Including the radiative cooling in the dynamics is essential, because it creates an important difference in the resulting spectra. Indeed, in the infrared, which probes radiation from the accretion flow very close to the $\mathrm{BH}$, the predicted emission is two orders of magnitude higher compared to that without cooling.

\section{Conclusion}

These preliminary results are thus very interesting and exciting. We are finishing an exploration of parameter space in $2 \mathrm{D}$, and then will choose some of those cases for $3 \mathrm{D}$ followup.

\section{References}

Peter Anninos, P. Chris Fragile, Jay D Salmonson 2005, The Astrophysical Journal, 635, 723 